\title{
Single-Site Robotic Myomectomy without Accessory Instrument Compared with Two-Port Laparoscopic Myomectomy: A Propensity Score Matching Analysis
}

\author{
Ji-Hye Kim Eun-Ju Lee
}

Department of Obstetrics and Gynecology, Chung-Ang University College of Medicine, Seoul, Republic of Korea

\section{Keywords}

Single-site robotic surgery · Uterine myomectomy ·

Perioperative outcome

\begin{abstract}
Objectives: Despite the advantages of robotic technology, single-site robotic myomectomy (SSRM) without an accessory instrument is limited by a restricted range of motion, weaker suturing of a thick myometrium, and non-articulating instruments. We present our novel gradual turning out method (GTOM) of SSRM and our assessment of its feasibility and safety by comparing its perioperative outcomes with those of two-port laparoscopic myomectomy (LM). Design: A retrospective cohort case-control study was carried out. Methods: This study included consecutive 46 patients who underwent SSRM for intramural myomas larger than $7 \mathrm{~cm}$, from 2016 to 2019. Subsequently, 46 patients who underwent LM were selected by 1:1 propensity score matching by controlling for age, body mass index, myoma number, myoma diameter, and the presence of sexual intercourse. The perioperative outcomes of the two groups were compared using a Mann-Whitney $U$ test and Fisher's exact test. The effect of covariates on operation time was analyzed using uni-
\end{abstract}

variable and multivariable linear regression. Results: SSRM was performed successfully with GTOM for myomas of up to $14 \mathrm{~cm}$ in the longest diameter, without conversion to laparotomy and intraoperative injuries. No differences between the groups were found in length of hospital stay, estimated blood loss, hemoglobin level decrease, transfusion rate, and postoperative pain, but operative time was significantly longer in the SSRM group than in the LM group ( $p<0.001)$. Larger myomas, location of the lower segment, and the operation method of SSRM were significantly associated with a longer operation time. Whereas operation time for myomas located at the anterior wall, singleton myomas, and myomas $<10 \mathrm{~cm}$ was significantly longer in the SSRM group than in the LM group, that for myomas at the posterior or lateral side of the uterus, multiple myomas, and myomas $\geq 10 \mathrm{~cm}$ did not differ significantly between the groups, indicating the advantage of SSRM for difficult myomectomy. Limitations: Retrospective nature of the study and limitation to a singlecenter study are the limitations of the study. Conclusions: Despite the lack of an accessory instrument, SSRM using the GOTM was feasible and safe as it yielded similar perioperative outcomes to those of LM.

(c) 2022 The Author(s). Published by S. Karger AG, Basel
Karger@karger.com www.karger.com/goi

Karger $\stackrel{\text { ' }}{5}$

GOPEN ACCESS
(C) 2022 The Author(s)

Published by S. Karger AG, Basel

This is an Open Access article licensed under the Creative Commons Attribution-NonCommercial-4.0 International License (CC BY-NC) (http://www.karger.com/Services/OpenAccessLicense), applicable to the online version of the article only. Usage and distribution for commercial purposes requires written permission.
Correspondence to:

Eun-Ju Lee, ejlee@cau.ac.kr 


\section{Introduction}

Uterine myomas are benign tumors that cause menorrhagia, dysmenorrhea, and urinary symptoms, which cause significant deterioration in quality of life. Surgical treatment is indicated in this condition. Abdominal myomectomy has been traditionally performed in women who desire to preserve their uterus. As surgical techniques evolved, laparoscopy became a substitute for laparotomy because of the well-known advantages of minimally invasive surgery [1]. However, laparoscopic myomectomy (LM) is still challenging on account of its technical complexity demonstrated by a steep learning curve [2]. Recently, the advent of robotic surgery has resulted in advantages such as three-dimensional stereoscopic vision and magnification, use of an articulating instrument, tremorfiltration, and reduced operation fatigue. Among robotic techniques, multi-port robot-assisted myomectomy (MRM) has been reported as a safe, minimally invasive surgery [3-6]. Accordingly, single-site robotic myomectomy (SSRM) is expected to provide further potential advantages, such as better cosmesis with higher patient acceptability, to umbilical single-site surgery. To date, only four studies on SSRM exist [7-10]; however, most of these studies used accessory instruments or additional ports.

In this study, we introduce a novel technique of SSRM, in which an accessory port is not required. The feasibility and safety of our technique were demonstrated by comparing its perioperative outcomes with those of conventional two-port LM.

\section{Materials and Methods}

\section{Patients}

This was a retrospective case-control analysis. We collected data from 360 patients who underwent myomectomy (SSRM, $n=$ 90; LM, $n=270$ ) by a single surgeon at an academic hospital from 2016 to 2019. All patients with indications for myomectomy were offered the choice of SSRM or LM. Forty-six consecutive cases of intramural myomas larger than $7 \mathrm{~cm}$ underwent SSRM. The SSRM and LM cohorts were matched 1:1 by propensity scoring, employed to minimize bias from a nonrandomized treatment sample.

We reviewed patients' records and summarized their clinical characteristics. The following parameters were recorded for each patient: age, height, weight, body mass index, education, occupation, continued sexual activity, menopause status, chief complaints, parity, and history of previous abdominal/pelvic surgeries. The number, location, and diameter of myomas were preoperatively evaluated by magnetic resonance imaging or ultrasonography. The diameters of myomas were compared on sagittal, axial, coronal, and oblique T2-weighted sequences of enhanced magnetic resonance imaging and the longest diameter was evaluated. Transabdominal ultrasonography with a full bladder, which was more useful than transvaginal scanning because of the large bulk of the uterus, was performed for all patients along with transvaginal or transrectal scanning. If available, magnetic resonance imaging was performed, and if not, ultrasonography was carried out.

Outcomes were obtained from the surgery and anesthesia records and included the weight of the removed myomas, the length of hospital stay, estimated blood loss, drop in the postoperative hemoglobin $(\mathrm{Hb})$ level, operative time, and postoperative complications. The operative time was defined as the time from skin incision to complete skin closure. The postoperative drop in $\mathrm{Hb}$ level was defined as the difference between Hb level in the morning of surgery and that at postoperative day 1 . In cases requiring transfusion during the surgery, the difference between $\mathrm{Hb}$ level in the morning of surgery and the lowest $\mathrm{Hb}$ level during surgery was calculated. The lowest preoperative $\mathrm{Hb}$ level accepted by anesthetists in our hospital is $9.0 \mathrm{~g} / \mathrm{dL}$, and cases with an $\mathrm{Hb}$ level $<8.0 \mathrm{~g} / \mathrm{dL}$ were transfused during the surgery. Adverse outcomes, including febrile morbidity, conversion to laparotomy, requirement for transfusion during the operation, and need for reoperation, were assessed. All patients were administered patient-controlled analgesia intravenously. Postoperative pain was defined as the use of rescue analgesics, injected when the patient complained of pain with a numerical rating scale score of $>4$. This retrospective chart review was approved by the Institutional Review Board of Chung-Ang University Hospital (IRB number: 2004-012-19313) on 10 June 2020.

\section{Surgical Procedure}

For SSRM, the robotic operation system, da Vinci Si platform (Intuitive Surgical, Inc., Sunnyvale, CA, USA) was used. A single surgeon, with experience of at least 20 cases of SSRM prior to case collection, performed the surgeries. A uterine manipulator was placed vaginally in all cases except in patients who denied being sexually active. A pneumoperitoneum was caused by placing a Veress needle (Step ${ }^{\text {TM }}$ Insufflation/Access Needle, Covidien, Mansfield, MA, USA) in the umbilicus. A vertical $2.0-2.5 \mathrm{~cm}$ umbilical opening was made via an open Hasson approach and the Gloveport (Nelis, Gyeonggi-do, Korea) was delivered into the incision (Fig. 1a-c). After the patient was placed in the Trendelenburg position, the abdominal and pelvic cavities were explored. Three laparoscopic gauzes were placed in the right paracolic gutter, near the right round ligament and posterior cul-de-sac. The suture materials were placed in the right paracolic gutter (Fig. 1d). Vasopressin at a concentration of $0.25 \mathrm{U} / \mathrm{mL}$ was infiltrated into the subcapsular area (Fig. 1e); a robotic system was then docked according to the manufacturer's recommendation; an $8.5 \mathrm{~mm}, 30^{\circ}$ stereo-endoscope was introduced through the robotic trocar, and two curved cannulas were introduced in the cross direction under direct visualization. Fenestrated bipolar forceps and a monopolar hook were inserted (Fig. 1f).

The gradual turning out method (GTOM) was followed, as detailed here. A horizontal incision (Fig. 1g) or semicircular incision for a large-sized mass at the serosa and surrounding myometrial shell was made using a monopolar hook. The myoma was held by stabbing at it with the hook and was extracted by peeling the surrounding pseudomembrane with bipolar forceps or vice versa (Fig. $1 \mathrm{~h}-\mathrm{j}$ ). This procedure was gently repeated in all directions of the incision, and the mass was gradually turned out. After the longest part of the mass was turned out, it was pushed aside, and the surrounding tissues were continuously coagulated and separated. The major part of the mass was removed from the uterus, and the 

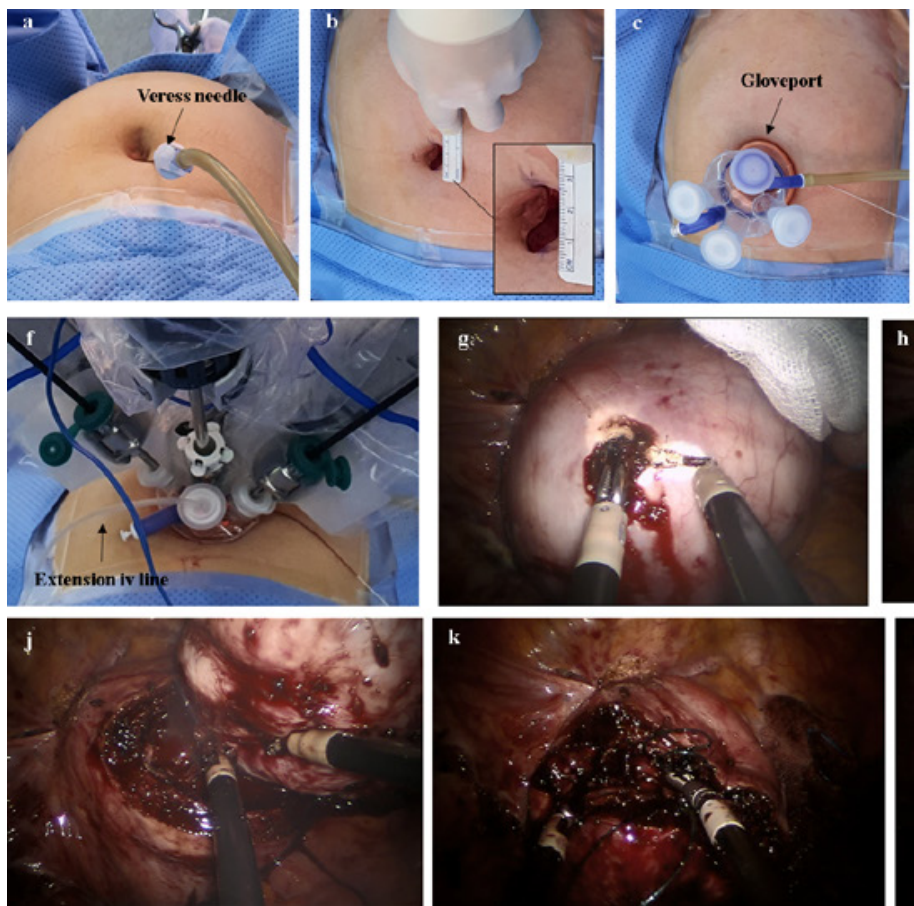

Fig. 1. The procedure of SSRM. a A pneumoperitoneum with a Veress needle is established. b $2.0-2.5-\mathrm{cm}$ skin incision, as measured using a sterile ruler, is made. c The Gloveport is placed in the umbilical opening. d Gauzes and suture material are placed at the right paracolic gutter. e Vasopressin is infiltrated into the subcapsular area of myoma. $\mathbf{f}$ The robotic docking status with the placement of camera, two curved trocars, and intravenous extension line is shown. $\mathbf{g}$ An incision at the serosa and surrounding myo-

stump was finally separated. The specimen was placed in the right paracolic gutter. The monopolar hook was exchanged for a EndoWrist ${ }^{\circledR}$ Si wristed needle driver, and the uterine myometrium was reapproximated layer by layer for two more layers using continuous running 1-0 V Loc sutures (Covidien, Dublin, Ireland) (Fig. 1k). The serosa was repaired in a running "baseball" fashion (Fig. 11). The specimen was retrieved into the endopouch and brought up to the umbilical opening by morcellation with a scalpel (Fig. $1 \mathrm{~m}$ ). Figure 2 shows the GTOM in another case. For LM, the method previously reported was performed [11].

\section{Statistical Analysis}

The propensity score was computed using age, body mass index, number and diameter of myomas, and the continued engagement in sexual intercourse as controlling covariates, and 1:1 sample matching was performed using the nearest matching neighbor method. The matched data were used to analyze differences in surgical outcomes between SSRM and LM.

The sample number and percentage were computed for categorical variables. Continuous variables were presented as means \pm standard deviations or medians (interquartile ranges), according to their normality, which was estimated using Lilliefors (Kolmogorov-Smirnov) and Shapiro-Wilk normality tests. For the propensity score-matched sample data, the mean differences in
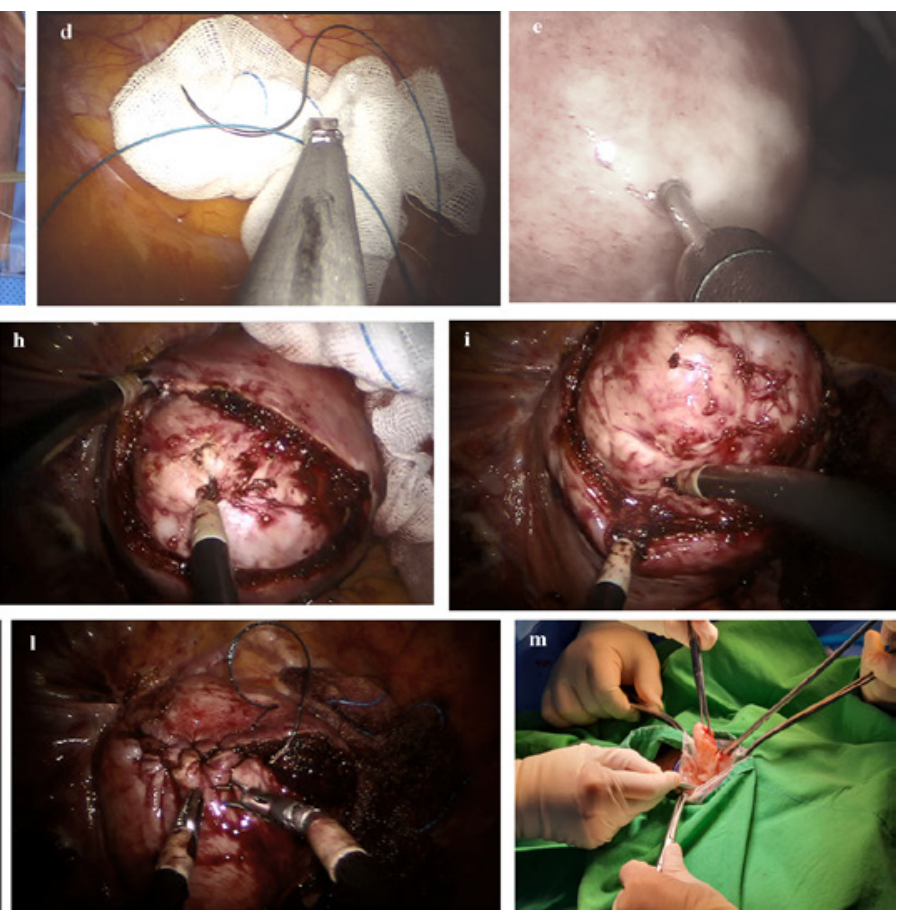

metrial shell is made with a monopolar hook. $\mathbf{h}, \mathbf{i}$ The myoma is held by stabbing it with the monopolar hook and peeling the pseudomembrane with bipolar forceps. $\mathbf{j}$ The myoma is pushed aside and the surrounding tissues are finally coagulated and separated. k The myometrial layer is sutured with 1-0 V Loc suture. I The suturing along the serosal layer is made in a running baseball fashion. $\mathbf{m}$ The specimen is retrieved into the endopouch and morcellated with a scalpel.

continuous surgical outcomes such as length of hospital stay, estimated blood loss, postoperative Hb level drop, and operative times between those who underwent SSRM and LM were analyzed using a Student's $t$ test or Mann-Whitney $U$ test, by considering normally distributed variables. The association between operation method and binary surgical outcomes such as transfusion during surgery and postoperative pain in $12 \mathrm{~h}$ was analyzed using a Fisher's exact test. To analyze the effect of covariates such as myoma size, multiplicity, location, and operation method on operative time, a linear regression analysis was performed. $\mathrm{R}$ language version 3.3.3 (R Foundation for Statistical Computing, Vienna, Austria) and T\&F software version 3.0 (YooJin BioSoft, Korea) were used for all statistical analyses. Statistical significance was defined as $p$ value $<0.05$.

\section{Results}

There were no cases of conversion to laparotomy, need for an additional port, intraoperative injury, and reoperation among the 92 patients included in the analyses. As seen in Table 1, baseline characteristics between the two 

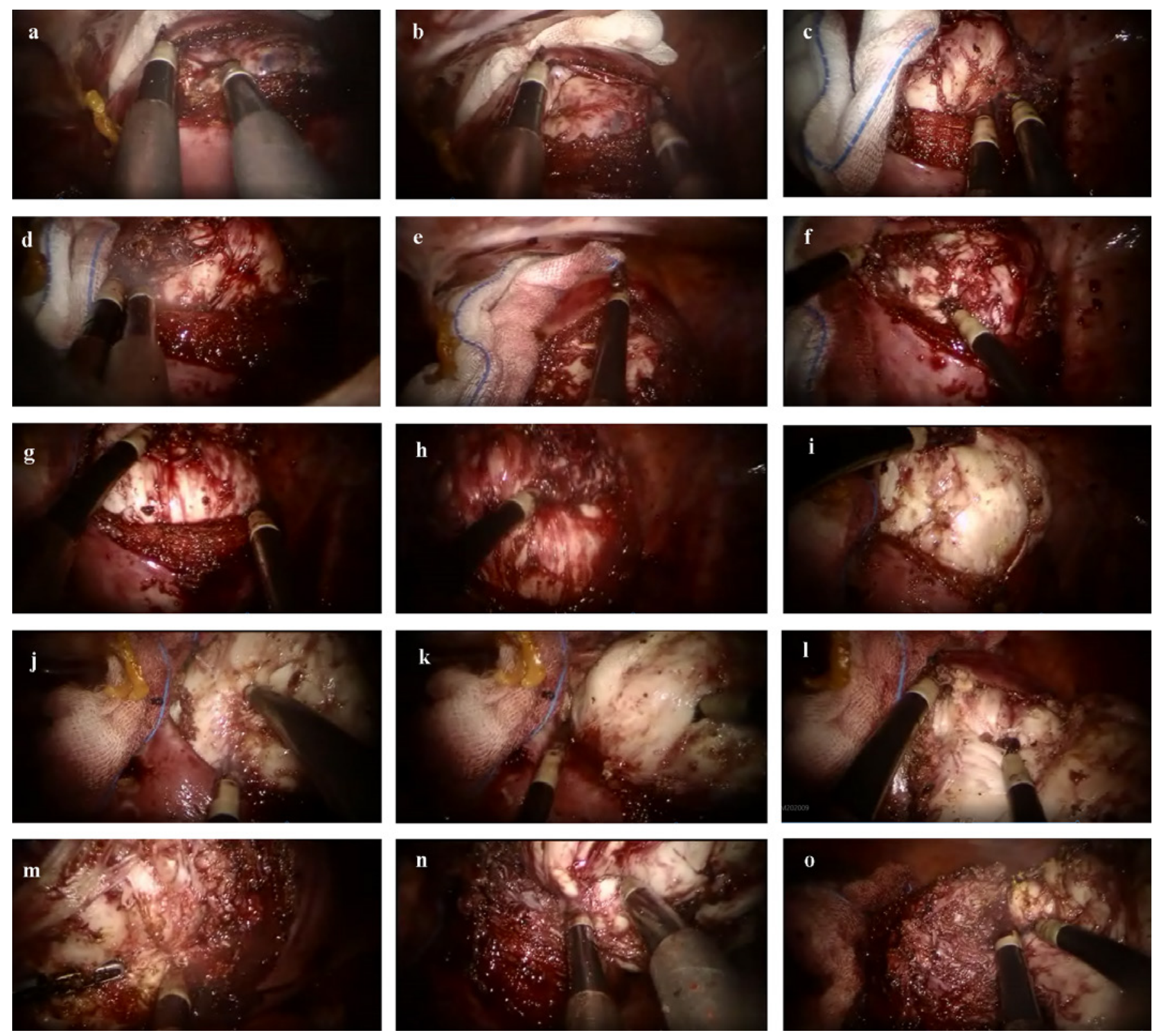

Fig. 2. The gradual turning out method. a-d Gentle peeling surrounding the pseudomembrane is achieved by successively pushing forward with bipolar forceps, fixing the myoma by stabbing with a monopolar hook, and repeating this procedure successively in the 12 o'clock, 3 o'clock, 6 o'clock, and 9 o'clock direction. e-i Repeating the procedure enables turning out the myoma mass gradually. $\mathbf{j}-\mathbf{m}$ Once the longest diameter of the mass is turned out, the myometrial opening shrinks. $\mathbf{n}, \mathbf{o}$ The mass is pushed aside and the remaining attached tissues are continuously coagulated and separated; the major part of mass is removed from the uterus, and the stump is finally cut. groups did not differ. Among 3 patients with menopause who desired to preserve their uterus, 1 had a palpable mass and 2 had urinary frequency. The majority of patients presented with menorrhagia (47.8\%) and dysmenorrhea (31.6\%). Thirty-four (37\%) patients had a history of previous intra-abdominal surgery, including caesarean section, laparoscopic appendectomy, laparoscopic salpingectomy, laparoscopic unilateral salpingo-oophorectomy, laparoscopic cholecystectomy, and LM. 
Table 1. Characteristics of patients in the SSRM and LM groups

\begin{tabular}{|c|c|c|c|c|}
\hline Variable & Total $(N=92)$ & $\operatorname{SSRM}(N=46)$ & $\operatorname{LM}(N=46)$ & $p$ value \\
\hline Age (mean $\pm S D$ ), years & $39.45 \pm 7.04$ & $39.37 \pm 6.71$ & $39.52 \pm 7.43$ & $0.918^{*}$ \\
\hline Height (mean \pm SD), $\mathrm{cm}$ & $160.89 \pm 5.51$ & $161.17 \pm 5.18$ & $160.61 \pm 5.87$ & $0.626^{*}$ \\
\hline Weight, median (IQR), kg & $59(53.25-64.75)$ & $59(54-68.5)$ & $58.5(52-63.25)$ & $0.375^{* *}$ \\
\hline BMI, median (IQR), $\mathrm{kg} / \mathrm{m}^{2}$ & $23(20-25.75)$ & $22.5(20-26.25)$ & $23(21-25.25)$ & $0.919 * *$ \\
\hline \multicolumn{5}{|l|}{ Education, $n(\%)$} \\
\hline Middle school & $2(2.2)$ & $0(0)$ & $2(4.3)$ & \multirow{3}{*}{$0.497^{\dagger}$} \\
\hline High school & $13(14.1)$ & $6(13)$ & $7(15.2)$ & \\
\hline Bachelor's degree & 77 (83.7) & $40(87)$ & $37(80.4)$ & \\
\hline \multicolumn{5}{|l|}{ Occupation, $n(\%)$} \\
\hline Employed & $60(65.2)$ & $33(71.7)$ & $27(58.7)$ & \multirow{2}{*}{$0.189^{t+}$} \\
\hline Unemployed & $32(34.8)$ & $13(28.3)$ & $19(41.3)$ & \\
\hline \multicolumn{5}{|l|}{ Parity, $n(\%)$} \\
\hline Nulliparous & $58(63)$ & $30(65.2)$ & $28(60.9)$ & \multirow{2}{*}{$0.666^{t+}$} \\
\hline Multiparous & $34(37)$ & $16(34.8)$ & $18(39.1)$ & \\
\hline \multicolumn{5}{|l|}{ Sexual experience, $n(\%)$} \\
\hline No & $74(80.4)$ & $36(78.3)$ & $38(82.6)$ & \multirow{2}{*}{$0.599^{\dagger \dagger}$} \\
\hline Yes & 18 (19.6) & $10(21.7)$ & $8(17.4)$ & \\
\hline \multicolumn{5}{|l|}{ Menopause status, $n(\%)$} \\
\hline No & $89(96.7)$ & $45(97.8)$ & $44(95.7)$ & \multirow{2}{*}{$>0.999^{\dagger}$} \\
\hline Yes & $3(3.3)$ & $1(2.2)$ & $2(4.3)$ & \\
\hline \multicolumn{5}{|l|}{ Chief complaint, $n$ (\%) } \\
\hline Menorrhagia & $44(47.8)$ & $25(54.3)$ & $19(41.3)$ & \multirow{4}{*}{$0.801^{\dagger}$} \\
\hline Dysmenorrhea & $29(31.6)$ & $13(28.3)$ & $16(34.8)$ & \\
\hline Palpable mass & $14(15.2)$ & $5(10.9)$ & $9(19.6)$ & \\
\hline Urinary frequency & $5(5.4)$ & $3(6.5)$ & $2(4.3)$ & \\
\hline \multicolumn{5}{|c|}{ Previous abdominal surgery, $n(\%)$} \\
\hline No & $58(63)$ & $32(69.6)$ & $26(56.5)$ & \multirow{2}{*}{$0.195^{t+}$} \\
\hline Yes & $34(37)$ & $14(30.4)$ & $20(43.5)$ & \\
\hline
\end{tabular}

SSRM, single-site robotic myomectomy with accessary port; LM, two-port laparoscopic myomectomy; SD, standard deviation; IQR, interquartile range; $\mathrm{BMI}$, body mass index. * Independent two sample $T$ test. ${ }^{* *}$ Mann-Whitney $U$ test. ${ }^{\dagger}$ Fisher's exact test. ${ }^{\dagger+} X^{2}$ test.
The characteristics of uterine myoma did not differ between the two groups (Table 2). Fifteen of the 92 patients $(16.3 \%)$ had a myoma greater than $10 \mathrm{~cm}$. The largest myoma was $14 \mathrm{~cm}$ and $16.9 \mathrm{~cm}$ in the SSRM and LM groups, respectively. In 61 patients (66.3\%), the myomas were located in the anterior wall or the fundus. A patient in the SSRM group had a low-segmentally located myoma.

No patient had postoperative fever. Perioperative outcomes including length of hospital stay, estimated blood loss, postoperative $\mathrm{Hb}$ level drop, need for transfusion during surgery, and pain (defined as a numeric rating scale score $\geq 4$ ) in the postoperative period, were similar in both groups (Table 3). The operative time was significantly longer in the SSRM group than that in the LM group $(p<0.001)$. Four patients in the SSRM group, who had a preoperative $\mathrm{Hb}$ level $<9.0 \mathrm{~g} / \mathrm{dL}$, required blood transfusion during surgery and received $0.5 \mathrm{~L}$ of blood regardless of the amount of blood loss. Nine patients needed additional analgesics within $12 \mathrm{~h}$ after surgery, but not thereafter. The characteristics of postoperative pain were upper abdominal discomfort and right shoulder pain, suggesting pneumoperitoneum-related pain.

We investigated parameters that influenced operative time (Table 4). In the univariable analysis, larger sized myomas, single myomas, a lower segmental location of the myoma, and SSRM method were significantly associated with a longer operative time $(p=0.007, p=0.024, p=0.005$, and $p=0.008$, respectively). In the multivariable analysis, the multiplicity variable was excluded because of multicollinearity, and larger sized myomas, a lower segmental location, and SSRM method were independently associated with a longer surgery time $(p=0.034, p=0.031$, and $p=$ 0.008 , respectively). We then assessed how these factors differed between the two groups (Table 5). The operative time for myomas located at the anterior wall or fundus, was significantly longer in the SSRM group than in the LM group ( $p=0.016)$. However, this was not the case, for those located at the posterior or lateral wall. The operative time for 
Table 2. Characteristics of uterine myomas in the SSRM and LM groups

\begin{tabular}{|c|c|c|c|}
\hline \multirow[t]{2}{*}{ Variable } & \multicolumn{2}{|l|}{$N(\%)$} & \multirow[t]{2}{*}{$p$ value } \\
\hline & $\operatorname{SSRM}(N=46)$ & $\operatorname{LM}(N=46)$ & \\
\hline Diameter, median (IQR), cm & $8.3(7.1-9.5)$ & $8.4(7.6-9.5)$ & $0.674^{*}$ \\
\hline \multicolumn{4}{|l|}{ Size of myoma, cm } \\
\hline$<10$ & $38(82.6)$ & $39(84.8)$ & \multirow{2}{*}{$0.778^{* *}$} \\
\hline$\geq 10$ & $8(17.4)$ & $7(15.2)$ & \\
\hline \multicolumn{4}{|l|}{ Method of size measurement } \\
\hline MRI & $19(41.3)$ & $16(34.8)$ & \multirow{2}{*}{$0.519 * *$} \\
\hline Ultrasonography & $27(58.7)$ & $30(65.2)$ & \\
\hline \multicolumn{4}{|l|}{ Multiplicity } \\
\hline Single & $31(67.4)$ & $33(71.7)$ & \multirow{2}{*}{$0.650 * *$} \\
\hline Multiple & $15(32.6)$ & $13(28.3)$ & \\
\hline \multicolumn{4}{|l|}{ Location } \\
\hline Anterior/fundus & $32(69.6)$ & $29(63)$ & \multirow{4}{*}{$0.589^{\dagger}$} \\
\hline Posterior & 9 (19.6) & $14(30.4)$ & \\
\hline Lateral & $4(8.7)$ & $3(6.5)$ & \\
\hline Low segment & $1(2.2)$ & $0(0.0)$ & \\
\hline Weight of surgical specimen, median (IQR), g & $175.5(119.8-262.5)$ & $168(100.8-253.0)$ & $0.380^{*}$ \\
\hline
\end{tabular}

SSRM, single-site robotic myomectomy with accessory port; LM, two-port laparoscopic myomectomy; IQR, interquartile range; MRI, magnetic resonance imaging. ${ }^{*}$ Mann-Whitney $U$ test. ${ }^{* *} X^{2}$ test. ${ }^{\dagger}$ Fisher's exact test.

Table 3. Comparison of perioperative outcomes between the SSRM and LM groups

\begin{tabular}{lllr}
\hline Variable & SSRM $(N=46)$ & LM $(N=46)$ & $p$ value \\
\hline Length of hospital stay, median (IQR), days & $4(4-4)$ & $4(4-4)$ & $0.960^{*}$ \\
EBL, median (IQR), mL & $100(50-200)$ & $150(87.5-250)$ & $0.227^{*}$ \\
Hb drop, median (IQR), g/dL & $2.0(1.50-2.75)$ & $1.85(1.3-2.5)$ & $0.265^{*}$ \\
OP time, median (IQR), min & $190(147.5-251.3)$ & $145(110-188.8)$ & $<0.001^{*}$ \\
Transfusion during op, $n(\%)$ & & $46(100)$ & $0.117^{\dagger}$ \\
$\quad$ No & $42(91.3)$ & $0(0)$ & $0.485^{\dagger}$ \\
$\quad$ Yes & $4(8.7)$ & $43(93.5)$ & $3(6.5)$ \\
$\quad$ \\
$\quad$ Postoperative pain within $12 \mathrm{~h}, n(\%)$ & $40(87.0)$ & $6(13.0)$ & \\
$\quad$ Yes &
\end{tabular}

SSRM, single-site robotic myomectomy with accessory port; LM, two-port laparoscopic myomectomy; IQR, interquartile range; EBL, estimated blood loss; $\mathrm{Hb}$, hemoglobin; OP, operation. ${ }^{*}$ Mann-Whitney U test. ${ }^{\dagger}$ Fisher's exact test.

a myoma at the lower segment was $430 \mathrm{~min}$, which was longer than the upper limit of the interquartile range of the operative time for myomas located at other sites. The operative time for single myomas was significantly longer in the SSRM group than in the LM group $(p=0.002)$, whereas that for multiple myomas was not significantly different between the two groups. The operative time for myomas less than $10 \mathrm{~cm}$ was longer in the SSRM than in the LM group $(p<0.001)$, whereas that for myomas greater than $10 \mathrm{~cm}$ was not significantly different between the groups.

Single-Site Robotic Myomectomy with a Novel Gradual Turning Out Method

\section{Discussion}

The results of this study showed that SSRM without an accessory port and using the GTOM does not require conversion to laparotomy, is not associated with intraoperative injuries, and has perioperative outcomes comparable to those of LM. Therefore, this surgical modality is safe and feasible.

For myomectomy, MRM is commonly used, and the majority of studies have compared MRM and LM [3-6]. 
Table 4. Clinical parameters associated with longer operative time

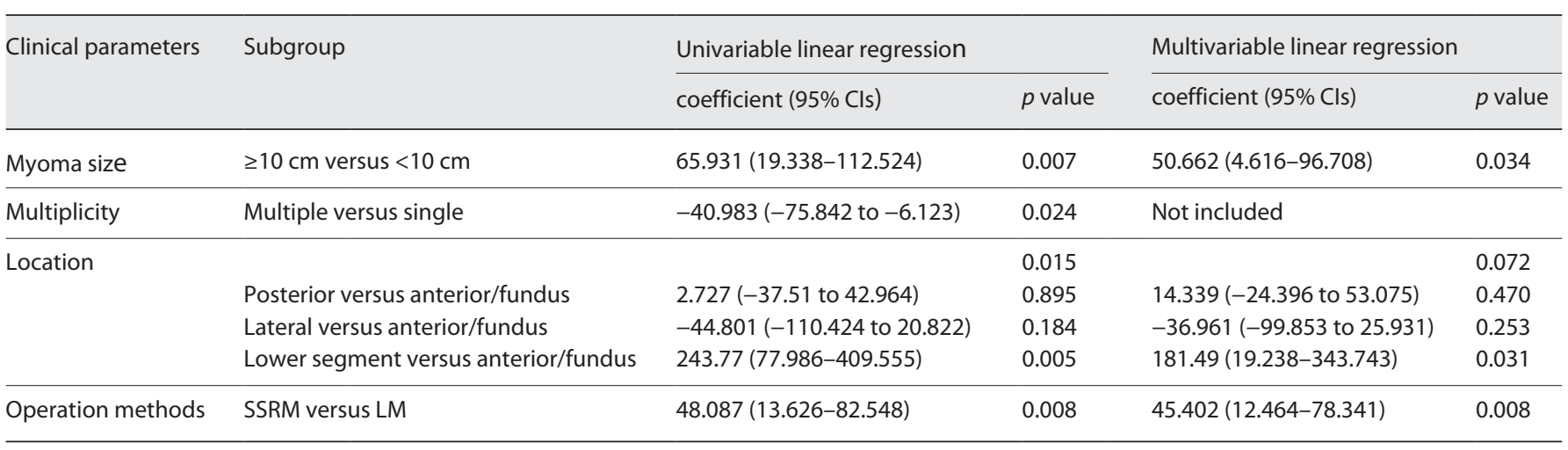

$\mathrm{Cl}$, confidence interval; SSRM, single-site robotic myomectomy with accessory port; LM, two-port laparoscopic myomectomy.

Table 5. Comparison of operation time according to clinical parameters associated with longer operation time between the SSRM and LM groups

\begin{tabular}{|c|c|c|c|}
\hline \multirow[t]{2}{*}{ Clinical parameters } & \multicolumn{2}{|c|}{$\begin{array}{l}\text { Operation time, median } \\
\text { (interquartile range) }\end{array}$} & \multirow[t]{2}{*}{$p$ value* } \\
\hline & SSRM $(N=46)$ & $\operatorname{LM}(N=46)$ & \\
\hline \multicolumn{4}{|l|}{ Location } \\
\hline Anterior/fundus & $190(155-249)$ & $150(105-220)$ & 0.016 \\
\hline Posterior & $200(153-305)$ & 158 (118-189) & 0.108 \\
\hline Lateral & $138(128-204)$ & $140(85-140)$ & 0.854 \\
\hline Lower segment & $430(430-430)$ & - & - \\
\hline \multicolumn{4}{|l|}{ Multiplicity } \\
\hline Single & $200(150-250)$ & $140(105-180)$ & 0.002 \\
\hline Multiple & $175(140-275)$ & $150(122.5-245)$ & 0.259 \\
\hline \multicolumn{4}{|l|}{ Myoma size } \\
\hline$<10 \mathrm{~cm}$ & $178(148-225)$ & $140(100-180)$ & $<0.001$ \\
\hline$\geq 10 \mathrm{~cm}$ & $260(164-333)$ & $175(160-270)$ & 0.536 \\
\hline
\end{tabular}

SSRM, single-site robotic myomectomy with accessory port; LM, two-port laparoscopic myomectomy. * Mann-Whitney U test.

Two meta-analyses concluded no inferiority and no significant short-term surgical benefits of MRM over LM. In contrast, SSRM has its own challenges and its safety and feasibility have been reported in limited studies [7-10]. A previous report that compared the surgical outcomes of SSRM and MRM showed that SSRM is equivalent to MRM [9]. To the best of our knowledge, this is the first study to compare SSRM and LM.

In this study, propensity score matching was adopted to eliminate the inherent bias of retrospective studies. In a retrospective study, subjects in two groups differ in terms of confounders, and differences in outcomes may reflect differences in baseline conditions, rather than a real treatment effect. Matching patients with a similar estimated propensity score, which is defined as the probability of each subject being assigned to a group of interest for comparison purposes, creates approximate balance for all the confounders, and differences in outcomes give unbiased estimates of the treatment effect. Therefore, we believe that our study made meaningful comparisons between SSRM and LM using propensity score matching.

SSRM has some drawbacks compared to MRM. These include a restricted range of motion due to the longcurved trocar, especially in cases of large-sized myomas; less powered suturing of the thick myometrium due to the semirigid shaft of the needle driver, and the inability to use multifunctional energy devices, or non-articulating instruments other than needle drivers [12]. To overcome these limitations, a larger umbilical incision was made, an accessory port was used, and laparoscopic technology was incorporated $[9,10,13,14]$. However, these adjustments may neutralize the advantages of single-port surgery. We therefore introduced our technique of SSRM. First, we separated the myoma from the pseudomembrane using the GTOM. Although this approach takes more time than direct myoma traction with a screw or forceps, bleeding is well controlled and an additional port for screw or forceps is not needed. Moreover, the efficacy of bleeding control, with this method, contributes to a clean operation field, which is critically required in minimally invasive surgery. Second, to reserve the working space of instruments, the uterus was fully pulled down and the longcurved trocars were properly pulled out. This is necessary in cases of large myomas, in which the distance between 
the instrument and target is too small for the manipulation of instruments. Accordingly, a parallel arrangement of the two instruments was made which did not interrupt the GTOM because the instruments worked forwardbackward or upward-downward, not sideways. We were concerned about the damage to the skin edge by excessive traction; however, the 20-25 mm incision did not result in any such injury. Third, an intravenous extension line was used to evacuate surgical smoke and often to suck blood in.

As expected, the operative time of SSRM was significantly longer than that of LM. This could be due to several factors. First, robotic surgery requires extra time for robotic docking and undocking and the substitution of instrumentation for the robotic arms [5]. Second, the inherent limitations of single-port surgery might contribute to the longer operative time. Third, the GTOM requires more time than direct traction with a screw or forceps. Despite the longer operative time, patients in the SSRM group did not present inferior surgical outcomes compared with those of patients who underwent LM, indicating that the average 45 -min difference in operative time did not increase perioperative complications.

In this study, the Gloveport was used, instead of the da Vinci (Intuitive Surgical, Sunnyvale, CA, USA) single-site port, which is relatively large in order to support a camera, two cannulas, and a 5-mm assisting port, and thereby requiring a $2.5-3-\mathrm{cm}$ skin incision. A smaller umbilical incision is generally preferred as it causes less pain, less chances of hernia, and better cosmesis. The Gloveport could potentially allow for a smaller umbilical incision and would not cause the surgeon discomfort.

This study had several limitations. It was a retrospective, single-center study with a limited sample size. However, by performing propensity score-matched analyses, we tried to minimize differences in patients' preoperative backgrounds. It is however necessary in the future, to conduct prospective cohort studies at multiple institutions to confirm our results.

\section{Statement of Ethics}

This retrospective chart review was approved and informed consent was waived by the Institutional Review Board of ChungAng University Hospital (IRB No. 2004-012-19313) on 10 June 2020 .

\section{Conflict of Interest Statement}

The authors declare no conflict of interests.

\section{Funding Sources}

This study was supported by the Basic Science Research Program through the National Research Foundation of Korea funded by the Ministry of Education (2020R1A2C1003536).

\section{Author Contributions}

Lee E.J. designed the study; Lee E.J. and Kim J.H. interpreted the results and drafted the manuscript. All authors are responsible for the article and have taken part in writing, reviewing, and revising the contents of the article.

\section{Data Availability Statement}

All data generated or analyzed during this study are included in this article. Further inquiries can be directed to the corresponding author.

\section{References}

1 Jin C, Hu Y, Chen XC, Zheng FY, Lin F, Zhou $\mathrm{K}$, et al. Laparoscopic versus open myomectomy - a meta-analysis of randomized controlled trials. Eur J Obstet Gynecol Reprod Biol. 2009;145:14-21.

2 Dubuisso JB, Fauconnier A, Babaki-Fard K, Chapron C. Laparoscopic myomectomy: a current view. Hum Reprod Update. 2000;6: 588-94.

3 Iavazzo C, Mamais I, Gkegkes ID. Robotic assisted versus laparoscopic and/or open myomectomy: systematic review and meta-analysis of the clinical evidence. Arch Gynecol Obstet. 2016;294:5-17.
4 Pundir J, Pundir V, Walavalkar R, Omanwa K, Lancaster G, Kayani S. Robotic-assisted laparoscopic vs abdominal and laparoscopic myomectomy: systematic review and metaanalysis. J Minim Invasive Gynecol. 2013;20: 335-45.

5 Nezhat C, Lavie O, Hsu S, Watson J, Barnett O, Lemyre M. Robotic-assisted laparoscopic myomectomy compared with standard laparoscopic myomectomy - a retrospective matched control study. Fertil Steril. 2009;91: 556-9.
6 Park SY, Kin J, Jeong K, Jung SI, Hur YM, Cho $\mathrm{EH}$, et al. Clinical experience of robotic myomectomy for fertility preservation using preoperative magnetic resonance imaging predictor. Obstet Gynecol Sci. 2020;63:726-34.

7 Gargiulo AR, Bailey AP, Srouji SS. Robot-assisted single-incision laparoscopic myomectomy: initial report and technique. J Robot Surg. 2013;7:137-42.

8 Lewis EI, Srouji SS, Gargiulo AR. Robotic single-site myomectomy: initial report and technique. Fertil Steril. 2015;103:1370-7.e1.
Single-Site Robotic Myomectomy with a Novel Gradual Turning Out Method 
9 Moawad GN, Tyan P, Paek J, Tappy EE, Park $\mathrm{D}$, Choussein $\mathrm{S}$, et al. Comparison between single-site and multiport robot-assisted myomectomy. J Robot Surg. 2019;13:757-64.

10 Choi EJ, Rho AM, Lee SR, Jeong K, Moon HS. Robotic single-site myomectomy: clinical analysis of 61 consecutive cases. J Minim Invasive Gynecol. 2017;24:632-9.
11 Shin IW, Park H, Kang H, Jung YJ, Lee EJ. Hybrid laparoendoscopic single-site surgery using a 2 -mm mini-laparoscopic instrument versus conventional three-port laparoscopy for gynecologic adnexal diseases: a prospective randomized trial. Gynecol Obstet Invest. 2019;84:495-502.

12 Iavazzo C, Minis EE, Gkegkes ID. Single-site port robotic-assisted hysterectomy: an update. J Robot Surg. 2018;12:201-13.
13 Scheib SA, Fader AN. Gynecologic robotic laparoendoscopic single-site surgery: prospective analysis of feasibility, safety, and technique. Am J Obstet Gynecol. 2015;212: 179-8.

14 Lee NH, Lee SH, Kim WY. Comparison of reduced-port robotic surgery (RPRS) with conventional 2 port laparoscopy for myomectomy. Eur J Obstet Gynecol Reprod Biol. 2020;247:181-5. 